\title{
Monetary policy and heterogeneous expectations
}

\author{
William A. Branch • George W. Evans
}

Received: 7 May 2010 / Accepted: 10 May 2010 / Published online: 24 June 2010

(C) The Author(s) 2010. This article is published with open access at Springerlink.com

\begin{abstract}
This paper studies the implications for monetary policy of heterogeneous expectations in a New Keynesian model. The assumption of rational expectations is replaced with parsimonious forecasting models where agents select between predictors that are underparameterized. In a Misspecification Equilibrium agents only select the best-performing statistical models. We demonstrate that, even when monetary policy rules satisfy the Taylor principle by adjusting nominal interest rates more than one for one with inflation, there may exist equilibria with Intrinsic Heterogeneity. Under certain conditions, there may exist multiple misspecification equilibria. We show that these findings have important implications for business cycle dynamics and for the design of monetary policy.
\end{abstract}

Keywords Heterogeneous expectations - Monetary policy · Multiple equilibria · Adaptive learning

JEL Classification $\quad \mathrm{G} 12 \cdot \mathrm{G} 14 \cdot \mathrm{D} 82 \cdot \mathrm{D} 83$

We are grateful to Mordecai Kurz, an anonymous referee, and participants at the SITE Workshop on Diverse Beliefs for comments.

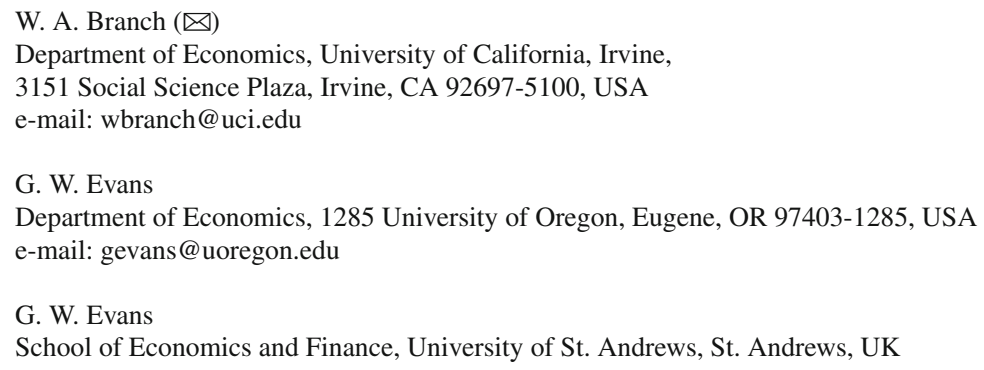




\section{Introduction}

The monetary policy literature has, for the most part, been developed within the rational expectations paradigm. One consequence of the assumption of rational expectations is that all agents in the economy hold homogeneous expectations formed with respect to the economy's true probability distribution. Recent studies of the implications for monetary policy of private agents following adaptive learning rules typically also maintain the assumption of homogeneity in expectation formation. There is, however, considerable empirical evidence that agents—households, firms, economists...- - hold heterogeneous beliefs.

This paper focuses on the implications for monetary policy of heterogeneous expectations. To do this we adopt the Misspecification Equilibrium framework of Branch and Evans (2006, 2007, 2009), itself an extension of Brock and Hommes (1997) to stochastic environments. Here we apply this approach to the New Keynesian model by assuming that agents select between forecasting models that differ by their explanatory variables. In the standard New Keynesian model of Woodford (2003), inflation and the output gap are driven by two exogenous processes corresponding to "demand" and "price" shocks. In a determinate model, the unique rational expectations equilibrium depends linearly on both demand and price shocks. We instead assume that agents favor parsimony in their forecasting models and, as a consequence, adopt underparameterized models: agents choose models that forecast the state based on demand shocks or price shocks, but not both. In a Misspecification Equilibrium, agents only select the best performing statistical models. In this sense, although agents hold misspecified beliefs, they will be optimal within the class of underparameterized models. A Misspecification Equilibrium is "in the spirit" of rational expectations equilibria by imposing important cross-equation restrictions while incorporating reasonable assumptions about the manner in which agents form their expectations.

Our approach is motivated by our earlier findings that even though all agents optimally select their underparameterized forecasting models, in equilibrium they may be distributed across all models (Branch and Evans 2006) or there may exist multiple equilibria (Branch and Evans 2007, 2009). Heterogeneity in beliefs and dispersion in forecasts is an empirical regularity (Branch 2004; Kurz and Motolese 2010). Thus, it is an important issue whether, and to what degree, monetary policy can affect the diversity of beliefs, and to what extent monetary policy should respond to this diversity. We assume that monetary policy can be described by a policy rule that adjusts interest rates in response to the current state of the economy. We find that, depending on the stochastic properties of the exogenous disturbances, there may exist multiple misspecification equilibria or equilibria exhibiting Intrinsic Heterogeneity.

These theoretical findings have several unique implications for business cycle dynamics and monetary policy. "Bad luck", in the form of more volatile and persistent price shocks, can turn an economy with a unique low volatility equilibrium into one with multiple equilibria. Real-time learning and dynamic predictor selection in such an economy will produce endogenous regime-switching volatility of the kind documented by Sims and Zha (2006), among others. However, if policy becomes increasingly aggressive in responding to inflation, as the Federal Reserve appears to have done during the mid 1980s, the economy will then coordinate on a unique equilibrium. 
We also examine the implications of heterogeneous expectations for a policymaker seeking to implement optimal discretionary policy. When monetary policy is set by a nominal interest rate rule that responds optimally to private-sector expectations, there exists a unique misspecification equilibrium. Interest rate rules of this form have been known to perform well when agents are homogeneous and use least-squares to update their forecasting models. Our results indicate that expectations-based policy rules also perform well when agents have heterogeneous expectations, even in circumstances in which multiple equilibria can arise under simple standard Taylor rules.

This paper proceeds as follows. In Sect. 2 we develop the New Keynesian model with diverse beliefs while Sect. 3.1 presents the main analytic results. Section 3.3 presents numerical examples of the number and nature of misspecification equilibria while Sect. 4 focuses on the business cycle and policy implications of heterogeneity. Section 5 concludes.

\section{Model}

\subsection{A New Keynesian model with diverse beliefs}

We develop our results within the framework of a standard "New Keynesian" model along the lines of Woodford (2003), where aggregate output and inflation are given by the following equations

$$
\begin{gathered}
x_{t}=\hat{E}_{t} x_{t+1}-\varsigma\left(i_{t}-\hat{E}_{t} \pi_{t+1}\right)+g_{t} \\
\pi_{t}=\beta \hat{E}_{t} \pi_{t+1}+\kappa x_{t}+u_{t}
\end{gathered}
$$

where we assume $g_{t}=\rho g_{t-1}+\varepsilon_{t}, u_{t}=\phi u_{t-1}+v_{t}, 0<\rho, \phi<1$. We allow for $g_{t}, u_{t}$ to be correlated. Here $x_{t}$ is the aggregate output gap, $\pi_{t}$ is the inflation rate, and $\hat{E}_{t}$ is a convex combination of expectations operators to be specified below. Note that under rational expectations $\hat{E}=E$.

Equations (1) and (2) constitute a New Keynesian model where conditional expectations have been replaced by a convex combination of boundedly rational expectations operators. The model shares the same reduced-form as the homogeneous expectations version of the New Keynesian model with either rational expectations or adaptive learning formulations along the lines of Bullard and Mitra (2002) and Evans and Honkapohja (2003). Branch and McGough (2009) derive these reduced-form equations from linear approximations to the optimal decision rules of a Yeoman-farmer economy extended to include two types of agents, ex ante identical except with respect to the manner in which they form expectations. The first Eq. (1) is an IS relation that describes the demand side of the economy. In an economy with homogeneous agents, rational or boundedly rational, it is a linear approximation to a representative agent's Euler equation. With agents heterogeneous in their expectation formation process, Eq. (1) is obtained by aggregating Euler equations across agents. The parameter 5 is the real interest elasticity of output. Equation (2) describes the aggregate supply 
relation. This is obtained by averaging each firms' pricing decisions, and as in the representative agent economy, $\kappa$ is the output elasticity of inflation.

Although Eqs. (1) and (2) share the same reduced-form as a representative agent economy, the key distinction here is that the diversity of beliefs leads the equilibrium processes for inflation and output to depend on the distribution of agents' expectations. This paper takes as given that these equations govern the economy, and is interested in the interaction of monetary policy and the equilibrium distribution of agents across (underparameterized) forecasting models.

\subsection{Monetary policy rules}

A fully specified model prescribes a nominal interest rate rule followed by the central bank. Throughout this paper we assume that monetary policy is set according to the nominal interest rate rule

$$
i_{t}=\chi_{\pi} \hat{E}_{t} \pi_{t}+\chi_{x} \hat{E}_{t} x_{t}
$$

According to this rule, policymakers respond to contemporaneous private sector expectations. We choose this form for several reasons. First, the robustness of monetary policies that respond to contemporaneous variables was stressed in Bullard and Mitra (2002). Second, the true values of $\pi_{t}$ and $x_{t}$ are not observable at the time policy is set and thus policies depending on them are not implementable, as argued by McCallum (1999). The contemporaneous expectations rule (3) was the preferred form in Bullard and Mitra (2002).

The precise form of the policy rule is actually not critical for the qualitative theoretical results presented in this paper. For example, we looked at rules where policymakers set interest rates in response to their own forecasts of contemporaneous inflation and output gap

$$
i_{t}=\chi_{\pi} \tilde{E}_{t} \pi_{t}+\chi_{x} \tilde{E}_{t} x_{t}
$$

where it may be the case that $\tilde{E} \neq \hat{E}$. We also looked at rules where they respond to contemporaneous state variables

$$
i_{t}=\chi_{\pi} \pi_{t}+\chi_{x} x_{t}
$$

which has a form close to the celebrated Taylor-rule, or based on expectations of future economic variables

$$
i_{t}=\chi_{\pi} \tilde{E}_{t} \pi_{t+1}+\chi_{x} \tilde{E}_{t} x_{t+1}
$$

With the latter rule, we examined cases where policymakers have rational expectations, their own boundedly rational forecasts, or respond to private-sector expectations. In each instance, the qualitative results are similar. In Sect. 4.3, we also consider optimal discretionary policy in which the policy rule depends in part on $\hat{E}_{t} \pi_{t+1}$ and $\hat{E}_{t} x_{t+1}$. 
The final reason we focus on rules of the form (3) is that they give a central role to private-sector expectations. This paper's primary purpose is to study the joint determination of monetary policy and the distribution of agents' beliefs. Policy rules of the form (3) deliver the sharpest results.

In each of the policy rules considered, nominal interest rates respond to aggregate state variables, and thus it is possible to represent the state of the economy in terms of an aggregate state vector $y=(x, \pi)^{\prime}$.

\subsection{Misspecification equilibrium}

In a minimum state variable rational expectations equilibrium, the law of motion for the state vector $y$ depends linearly on the exogenous processes for $g_{t}, u_{t}$. In this paper, we assume that agents favor parsimony in their forecasting model and instead select the best performing statistical models from the set of underparameterized forecasting models. Since there are only two exogenous variables, we assume that private sector agents underparameterize by selecting from two possible perceived laws of motion:

$$
\begin{aligned}
& P L M_{1}: y_{t}=b^{1} g_{t}+\eta_{t} \\
& P L M_{2}: y_{t}=b^{2} u_{t}+\eta_{t}
\end{aligned}
$$

where $\eta_{t}$ is a perceived exogenous white noise shock. This implies expectations of the form,

$$
\begin{array}{ll}
E_{t}^{1} y_{t}=b^{1} g_{t}, & E_{t}^{1} y_{t+1}=b^{1} \rho g_{t} \\
E_{t}^{2} y_{t}=b^{2} u_{t}, & E_{t}^{2} y_{t+1}=b^{2} \phi u_{t}
\end{array}
$$

Let $n$ denote the fraction of agents who use $P L M_{1}$. Then for any state variable $w$ we have $\hat{E} w=n E^{1} w+(1-n) E^{2} w$.

Substituting in the diverse beliefs along with the policy rule (3), the system can be written in the form

$$
y_{t}=A \hat{E}_{t} y_{t+1}+B \hat{E}_{t} y_{t}+D z_{t}
$$

where $z=(g, u)^{\prime}$ and the expressions for $A, B, D$ are provided in the Appendix. Plugging in private-sector expectations produces a reduced-form actual law of motion

$$
y_{t}=\xi_{1}(n) g_{t}+\xi_{2}(n) u_{t}
$$

where

$$
\begin{aligned}
& \xi_{1}(n)=n(\rho A+B) b^{1}+D e_{1}^{\prime} \\
& \xi_{2}(n)=(1-n)(\phi A+B) b^{2}+D e_{2}^{\prime}
\end{aligned}
$$

and $e_{i}, i=1,2$ is a unit row vector with the $i$ th component equal to one and zeros elsewhere. In the sequel, we will suppress the dependence of $\xi_{j}$ on $n$. 
Although private-sector agents are assumed to underparameterize their forecasting models, i.e. to hold restricted perceptions, in equilibrium we require that they forecast in a statistically optimal manner, in the sense that the forecast model parameters correspond to optimal linear projections. It follows then that the beliefs $b^{j}, j=1,2$ satisfy the following least-squares orthogonality conditions,

$$
\begin{aligned}
& E g_{t}\left(\xi_{1} g_{t}+\xi_{2} u_{t}-b^{1} g_{t}\right)=0 \\
& E u_{t}\left(\xi_{1} g_{t}+\xi_{2} u_{t}-b^{2} u_{t}\right)=0
\end{aligned}
$$

It follows that

$$
\begin{aligned}
& b^{1}=\xi_{1}+\xi_{2} r \\
& b^{2}=\xi_{2}+\xi_{1} \tilde{r}
\end{aligned}
$$

where $r=E g_{t} u_{t} / E g_{t}^{2}, \tilde{r}=E g_{t} u_{t} / E u_{t}^{2}$. Least squares orthogonality conditions like (5) or (6) appear frequently in the macroeconomics literature. For example, Sargent $(1999,2008)$ define a self-confirming equilibrium with respect to a very similar condition. Evans and Honkapohja (2001) and Sargent (2008) show that many learning models will converge to a set of parameters that satisfy orthogonality conditions like (5) and (6) rather than to their rational expectations values. The key feature of beliefs that satisfy orthogonality conditions like (5) or (6) are that within the context of their forecasting model, agents are unable to detect their model misspecification.

Combining equations, it is possible to solve for the reduced-form coefficients:

$$
\left[\begin{array}{l}
\xi_{1} \\
\xi_{2}
\end{array}\right]=\Delta^{-1}\left[\begin{array}{l}
D e_{1}^{\prime} \\
D e_{2}^{\prime}
\end{array}\right]
$$

where

$$
\Delta=\left[\begin{array}{cc}
I-n(\rho A+B) & -n(\rho A+B) r \\
-(1-n)(\phi A+B) \tilde{r} & I-(1-n)(\phi A+B)
\end{array}\right]
$$

We are now ready to define a Restricted Perceptions Equilibrium (RPE).

Definition Given exogenous processes for $g_{t}, u_{t}$ and given the proportion $n$ of agents using forecast model $j=1$, a Restricted Perceptions Equilibrium is a stochastic process $\left\{y_{t}\right\}$ of the form (4), where the coefficients satisfy (7).

Remark A unique RPE exists provided $\triangle^{-1}$ exists.

A general existence result is not available. However the following result holds for weakly correlated demand and price exogenous shocks and low levels of serial correlation.

Proposition 1 For values of $|r|,|\tilde{r}|,|\rho|,|\phi|$ sufficiently small, there exists a unique $R P E$ for all $0 \leq n \leq 1$. 
Because the model is self-referential, $b^{j}$ and $\xi_{j}$ are objects pinned down by the equilibrium. Similarly, we do not want to treat $n$ as a free parameter and we now make it endogenous. Thus, even though agents are assumed to forecast with misspecified models, there are still important equilibrium restrictions analogous to the restrictions obtained under fully rational expectations.

In order to determine $n$ endogenously, we need a metric for forecast success. Following Branch and Evans (2007), we assume that agents seek to minimize their forecast mean square error (MSE). Thus, we assume that agents rank their forecasting model according to

$$
E U^{j}=-E\left(y_{t+j}-E_{t}^{j} y_{t+j}\right)^{\prime} W\left(y_{t+j}-E^{j} y_{t+j}\right)
$$

where $W$ is a weighting matrix, which we set equal to the identity matrix. ${ }^{1}$ This is a natural metric since the prediction problem confronting agents, according to Eqs. (1) and (2), is to form one step ahead forecasts of the output gap and inflation.

Plugging in for private-sector expectations, in an RPE, the actual law of motion (4), leads to the MSE expressions

$$
\begin{aligned}
E U^{1}= & -E\left(\left(\xi_{2} \phi u_{t}-\xi_{2} \rho r g_{t}\right)+\xi_{1} \varepsilon_{t+1}+\xi_{2} v_{t+1}\right)^{\prime} \\
& \times W\left(\left(\xi_{2} \phi u_{t}-\xi_{2} \rho r g_{t}\right)+\xi_{1} \varepsilon_{t+1}+\xi_{2} v_{t+1}\right) \\
E U^{2}= & -E\left(\left(\xi_{1} \rho g_{t}-\xi_{1} \phi \tilde{r} u_{t}\right)+\xi_{1} \varepsilon_{t+1}+\xi_{2} v_{t+1}\right)^{\prime} \\
& \times W\left(\left(\xi_{1} \rho g_{t}-\xi_{1} \phi \tilde{r} u_{t}\right)+\xi_{1} \varepsilon_{t+1}+\xi_{2} v_{t+1}\right)
\end{aligned}
$$

The endogenous value for $n$ is assumed to depend on the relative forecast performance. Defining $F(n):[0,1] \rightarrow \mathbb{R}$ as $F(n)=E U^{1}-E U^{2}$, we can write the expression for relative forecast performance as

$$
F(n)=E g_{t}^{2}\left[\rho^{2}\left(\xi_{1}^{\prime} \xi_{1}-r^{2} \xi_{2}^{\prime} \xi_{2}\right)+2 \rho \phi\left(r \xi_{2}^{\prime} \xi_{2}-\tilde{r} \xi_{1}^{\prime} \xi_{1}\right) \tilde{r}+\phi^{2}\left(\tilde{r}^{2} \xi_{1}^{\prime} \xi_{1}-\xi_{2}^{\prime} \xi_{2}\right) Q\right]
$$

where $Q=E u_{t}^{2} / E g_{t}^{2}$. The relative forecasting performance depends on the distribution of beliefs because $\xi_{1}, \xi_{2}$ depend on $n$.

As in our earlier papers, we follow Brock and Hommes (1997) in assuming a multinomial logit (MNL) approach to predictor selection. The MNL approach has a long history in discrete decision making and is a natural way of introducing randomness in forecasting into a monetary model. Young (2004) argues that randomness in forecasting provides robustness against model uncertainty and flexibility in economic environments with feedback. Since our agents select their predictor from a discrete set of forecasting models, and are uncertain about which model forecasts best, the MNL

\footnotetext{
1 Our qualitative results are robust to alternative assumptions about how much relative weight agents place on inflation or output forecast errors. We note, however, that to remain consistent with the microfoundations of the model, the matrix $W$ should be diagonal (cf. Woodford 2003), and thus the identity matrix assumption is a natural choice.
} 
map is natural in this framework:

$$
n=\frac{\exp \left(\alpha E U^{1}\right)}{\exp \left(\alpha E U^{1}\right)+\exp \left(\alpha E U^{2}\right)}
$$

which can be re-written as

$$
n=\frac{1}{2}[\tanh \{\alpha F(n)\}+1] \equiv T_{\alpha}(n) .
$$

We remark that $T:[0,1] \rightarrow[0,1]$ is a continuous and well-defined function provided that an RPE exists. The parameter $\alpha$ is called the 'intensity of choice'. Since the MNL map derives from a random utility setting, finite values of $\alpha$ parameterize deviations from full utility maximization. The neoclassical limit arises for $\alpha \rightarrow \infty$ in the sense that in this case all agents select the best-performing statistical model.

In our theoretical results below, we focus on the case of large $\alpha$, because we are interested in whether monetary policy can affect the diversity of beliefs when agents are only subject to the restriction that they forecast with parsimonious models and the random component of utility is arbitrarily small. Finite values for $\alpha$ may perhaps be more realistic, but we take the "neoclassical" case as a benchmark and show that with this strong assumption, heterogeneity across models or across time is a theoretical possibility. Although the theoretical results restrict attention to the limiting case of large $\alpha$, in the numerical section below we illustrate the implications for finite $\alpha$.

That heterogeneity may arise even when each agent selects the best-performing model is surprising and was the main result in Branch and Evans (2006). Heterogeneity in the large $\alpha$ case can arise because the "best-performing model" is determined endogenously and depending on the nature of the feedback effect in the model, multiple predictors - each with distinct implications for agents' forecasts - may fare equally well within a misspecification equilibrium. Branch and Evans (2006) work with a cobweb model where there is negative feedback from expectations onto the state. With negative feedback agents have an incentive to deviate from a consensus forecasting model and so in equilibrium they will be distributed across diverse forecasting models. Even though the New Keynesian model has positive feedback the insight of this paper is that monetary policy that works through an interest rate reaction function may alter the feedback in such a way that heterogeneity will arise. The results below illustrate this insight.

It is important to emphasize that the feedback effects in this model - the RPE parameters, the distribution of agents, and the stochastic process for the state-are all jointly determined in an equilibrium. We are now ready to define our equilibrium concept.

Definition A Misspecification Equilibrium $n^{*}$ is a fixed point of the map $T: n^{*}=$ $T\left(n^{*}\right)$. 


\section{Results}

\subsection{Analytic results}

Since the MNL is a monotonic function of the relative predictor fitness measure, it should be clear from (8) that the number and nature of Misspecification Equilibria depends on the properties of $F(n)$. Using the arguments of Branch and Evans (2007), the following proposition characterizes the possible equilibria.

Proposition 2 Let $N_{\alpha}^{*}=\left\{n^{*} \mid n^{*}=T_{\alpha}\left(n^{*}\right)\right\}$ denote the set of Misspecification Equilibria. In the limit of large $\alpha, N^{*}$ has one of the following properties:

1. If $F(0)<0$ and $F(1)<0$ (Condition $P 0)$ then $n^{*}=0 \in N^{*}$.

2. If $F(0)>0$ and $F(1)>0$ (Condition Pl) then $n^{*}=1 \in N^{*}$.

3. If $F(0)<0$ and $F(1)>0($ Condition $P M)$ then $\{0, \hat{n}, 1\} \subset N^{*}$, where $\hat{n} \in(0,1)$ is such that $F(\hat{n})=0$.

4. If $F(0)>0$ and $F(1)<0$ (Condition $P)$ then $n^{*}=\hat{n} \in N^{*}$, where $\hat{n} \in(0,1)$ is such that $F(\hat{n})=0$.

In general, we do not know whether $F$ is monotonic, so we can not rule out the existence of additional equilibria besides those listed in Proposition 2. When Condition P0 or Condition P1 hold then either $n^{*}=0$ or $n^{*}=1$ is a Misspecification Equilibrium because in those instances agents would always want to select the demand disturbance or supply disturbance model when all other agents do as well. On the other hand, if Condition $\mathrm{P}$ holds then there is an incentive for an individual agent to deviate from a consensus model and instead Intrinsic Heterogeneity arises. Under Condition PM it follows that both $n^{*}=0$ and $n^{*}=1$ are Misspecification Equilibria. Thus, Condition PM is a sufficient condition for the existence of multiple equilibria. Under Condition PM there is also an interior equilibrium with agents distributed across both the demand and supply shock forecasting models. However, because $F(n)$ is a continuous function, Condition PM implies that whenever $F(n)$ is monotonic, we have $T^{\prime}(F(\hat{n}))>1$ and hence $\hat{n}$ is unstable under learning dynamics of the type we describe below. Under Condition $\mathrm{P}$, whenever $F(n)$ is monotonic then it crosses through zero from above, and we showed in Branch and Evans (2006) that a Misspecification Equilibrium with Intrinsic Heterogeneity is stable. Proposition 2 does not state the circumstances under which these conditions will arise. The model is multivariate and $F(0)$ and $F(1)$ depend in a complicated way on the parameters of the model. However, it is possible to demonstrate that conditions exist under which each of the various cases might arise.

Corollary 1 Conditions PO,P1,PM and P can each be satisfied for appropriate choices of structural parameters.

By focusing on the special case of weakly correlated demand and supply shocks, more precise results are available. In particular, the following result provides conditions under which either multiple Misspecification Equilibria or Intrinsic 
Heterogeneity arise. First, though, we define the following expressions:

$$
\begin{aligned}
& B_{0}=\left(\frac{\rho^{2}\left(1-\phi^{2}\right)}{\phi^{2}\left(1-\rho^{2}\right)}\right) \frac{\left(1+\kappa^{2}\right)\left[(1-\phi)(1-\beta \phi)+\kappa \varsigma\left(\chi_{\pi}-\phi\right)+\varsigma \chi_{x}(1-\beta \phi)\right]^{2}}{\varsigma^{2}\left(\chi_{\pi}-\phi\right)^{2}+\left(\varsigma \chi_{x}+1-\phi\right)^{2}} \\
& B_{1}=\left(\frac{\rho^{2}\left(1-\phi^{2}\right)}{\phi^{2}\left(1-\rho^{2}\right)}\right) \frac{\kappa^{2}+(1-\beta \rho)^{2}}{\left[(1-\beta \rho)^{2}(1-\rho)+\chi_{x} \varsigma(1-\beta \rho)+\kappa \varsigma\left(\chi_{\pi}-\rho\right)\right]^{2}}
\end{aligned}
$$

Proposition 3 For $r, \tilde{r}$ sufficiently small we have

1. Condition PO holds if

$$
\frac{\sigma_{v}^{2}}{\sigma_{\varepsilon}^{2}}>\max \left(B_{0}, B_{1}\right)
$$

2. Condition Pl holds if

$$
\frac{\sigma_{v}^{2}}{\sigma_{\varepsilon}^{2}}<\min \left(B_{0}, B_{1}\right)
$$

3. Condition PM holds if

$$
B_{0}<\frac{\sigma_{v}^{2}}{\sigma_{\varepsilon}^{2}}<B_{1}
$$

4. Condition P holds if

$$
B_{1}<\frac{\sigma_{v}^{2}}{\sigma_{\varepsilon}^{2}}<B_{0}
$$

This result illustrates that either multiple equilibria or Intrinsic Heterogeneity may arise under many different parameterizations of the model. Since $B_{0}, B_{1}$ do not depend on the variances of the exogenous white noise shocks, we are free to choose the relative ratio of these shocks to satisfy either of these conditions.

Proposition 4 Let $r, \tilde{r}$ be sufficiently small and assume $\alpha$ is large.

1. For $\chi_{\pi}$ and/or $\chi_{x}$ sufficiently large, depending on $\frac{\sigma_{v}^{2}}{\sigma_{\varepsilon}^{2}}$, either Condition $P$ holds, so that there exists a Misspecification Equilibrium with Intrinsic Heterogeneity, or Condition P0 holds, so that there exists a Misspecification Equilibrium with $n^{*}=0$.

2. For $\sigma_{v}^{2} / \sigma_{\varepsilon}^{2}$ sufficiently large, there exists a Misspecification Equilibrium at $n^{*}=0$.

3. For $\sigma_{v}^{2} / \sigma_{\varepsilon}^{2}$ sufficiently small, there exists a Misspecification Equilibrium at $n^{*}=1$.

Remark In case 1 of Proposition 4 Intrinsic Heterogeneity arises when $\frac{\sigma_{v}^{2}}{\sigma_{\varepsilon}^{2}}$ is less than a threshold value and $n^{*}=0$ arises when $\frac{\sigma_{v}^{2}}{\sigma_{\varepsilon}^{2}}$ is greater than the threshold. For further details, see the Appendix. 


\subsection{Intuition}

A simple example helps illustrate the intuition for why different equilibria may arise. The form of the actual law of motion (4) illustrates that the exogenous disturbances $g_{t}, u_{t}$ have direct and indirect effects via the feedback from expectations onto the state. The strength of the indirect effects are controlled by the parameters $\varsigma, \kappa, \beta, \chi_{\pi}, \chi_{x}$. The number and nature of Misspecification Equilibria depend on a balancing of these direct and indirect effects. To illustrate how multiple equilibria or intrinsic heterogeneity might arise, take the starkest parameterization of the model: suppose that demand and supply shocks are uncorrelated (i.e. $r=\tilde{r}=0$ ) and the indirect effects are weak, i.e. $\varsigma=0, \kappa=0, \beta=0$. Under these assumptions, the model (1) and (2) becomes

$$
\begin{aligned}
x_{t} & =E_{t} x_{t+1}+g_{t} \\
\pi_{t} & =u_{t}
\end{aligned}
$$

Suppose at first that $n=1$. The RPE parameters are

$$
\begin{aligned}
& \xi_{1}=(1 /(1-\rho), 0)^{\prime} \\
& \xi_{2}=(0,1)^{\prime}
\end{aligned}
$$

Agents within this RPE will have mean-square forecast errors $\frac{1}{1-\phi^{2}} \sigma_{v}^{2}$ for inflation and $\frac{1}{(1-\rho)^{2}} \sigma_{\varepsilon}^{2}$ for the output gap. Now consider a zero-mass agent deciding whether to deviate from the consensus and select the supply-shock model instead. Their beliefs, which satisfy their restricted perceptions orthogonality condition, are $b^{2}=(0,1)^{\prime}$. Their forecast errors are thus $\sigma_{v}^{2}$ for inflation and $\frac{1}{(1-\rho)^{2}\left(1-\rho^{2}\right)} \sigma_{\varepsilon}^{2}$ for the output gap. Whether $n=1$ can be a Misspecification Equilibrium depends on whether the zeromass agent has an incentive to deviate. That is, $n=1$ will be an equilibrium whenever

$$
\frac{\sigma_{v}^{2}}{\sigma_{\varepsilon}^{2}}<\frac{\rho^{2}\left(1-\phi^{2}\right)}{\phi^{2}\left(1-\phi^{2}\right)} \frac{1}{(1-\rho)^{2}}=B_{1}
$$

Now suppose that the economy is at $n=0$. The RPE parameters are

$$
\begin{aligned}
& \xi_{1}=(1,0)^{\prime} \\
& \xi_{2}=(0,1)^{\prime}
\end{aligned}
$$

Agents using the supply shock model will have forecast errors for inflation and output gap of $\sigma_{v}^{2}, \frac{1}{1-\rho^{2}} \sigma_{\varepsilon}^{2}$, respectively. An agent deciding to deviate from the consensus and forecast based on the demand shock model will have beliefs $b^{1}=(1,0)^{\prime}$ and forecast errors for inflation and output gap of $\frac{1}{1-\phi^{2}} \sigma_{v}^{2}, \sigma_{\varepsilon}^{2}$. Thus, $n=0$ will be an equilibrium whenever

$$
\frac{\sigma_{v}^{2}}{\sigma_{\varepsilon}^{2}}>\frac{\rho^{2}\left(1-\phi^{2}\right)}{\phi^{2}\left(1-\rho^{2}\right)}=B_{0}
$$


Since $B_{1}>B_{0}$ we have condition $P 0, P 1$ or $P M$, depending on the size of $\sigma_{v}^{2} / \sigma_{\varepsilon}^{2}$, and, stable Intrinsic Heterogeneity, i.e. Condition $P$, cannot arise. However, Intrinsic Heterogeneity can arise if there is negative feedback, e.g. from policy.

To see this we extend the special case just analyzed to allow for $\varsigma, \chi_{x}>0$. One can then show that $B_{1}<B_{0}$ when

$$
\left(1-\rho+\varsigma \chi_{x}\right)^{2}\left(1-\phi+\varsigma \chi_{x}\right)^{2}>\varsigma^{2} \phi^{2}
$$

which is satisfied for $\varsigma \chi_{x}$ sufficiently large. Intrinsic Heterogeneity arises in this case because of the negative impact on output of a strong policy response to expected output.

In most New Keynesian models a "Taylor principle" for monetary policy arises: in an interest rate rule, policy should move nominal rates more than one-for-one with inflation, i.e. $\chi_{\pi}>1$. The intuition behind the Taylor principle is that, by adjusting nominal interest rates by more than (expected) inflation, monetary policy will move the real rate in the desired direction. Under rational expectations and interest-rate rule (3), adhering to the Taylor principle ensures the existence of a unique rational expectations equilibrium. In the results presented in this paper, there is no corresponding Taylor principle for Misspecification Equilibria and, in fact, setting $\chi_{\pi}>1$ in some instances may lead to multiple equilibria. A brief extension of the current example illustrates how policy feedback via $\chi_{\pi}$ affects the number and nature of equilibria.

Suppose that $\beta=\kappa=r=\tilde{r}=\chi_{x}=0$. Now the model consists of the equations

$$
\begin{aligned}
x_{t} & =E_{t} x_{t+1}-\varsigma\left(\chi_{\pi} E_{t} \pi_{t}-E_{t} \pi_{t+1}\right)+g_{t} \\
\pi_{t} & =u_{t}
\end{aligned}
$$

Recall from above that when $\chi_{\pi}=0$, multiple equilibria can arise depending on $\sigma_{v}^{2} / \sigma_{\varepsilon}^{2}$. The question we ask is how values of $\chi_{\pi}$ affect this result. The expression for $B_{1}$ is unchanged, but now we have

$$
B_{0}=\left(\frac{\rho^{2}\left(1-\phi^{2}\right)}{\phi^{2}\left(1-\rho^{2}\right)}\right) \frac{(1-\phi)^{2}}{\varsigma^{2}\left(\chi_{\pi}-\phi\right)^{2}+(1-\phi)^{2}}
$$

Thus, $\chi_{\pi}>1$ does not necessarily imply a unique misspecification equilibrium. In fact, adjusting nominal interest rates in response to expected inflation may make multiple equilibria more likely. In this example, $\pi_{t}=u_{t}$ and by reacting to $u_{t}$ with the parameter $\chi_{\pi}$ the effect of $u_{t}$ on $x_{t}$ increases, making an $n=0$ equilibrium more likely to exist; that is, the "supply shock" predictor has greater explanatory power when policy feeds back on expected inflation.

This example nicely illustrates the equilibria that may arise in a New Keynesian model with misspecified beliefs. More plausible specifications for the indirect effects, and in particular the policy effects, lead to a rich set of equilibrium results. The following section illustrates the role that policy plays in determining the number and nature of Misspecification Equilibria. 

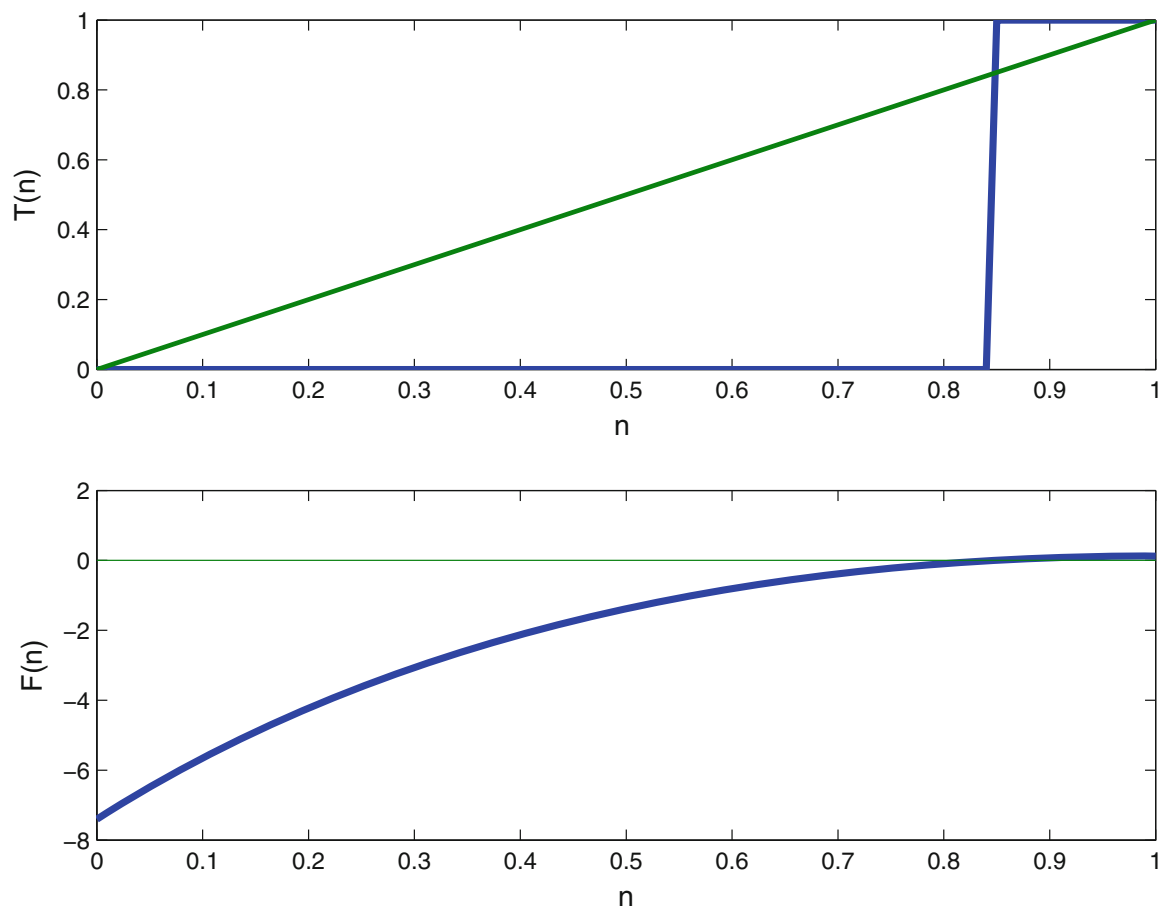

Fig. 1 Multiple equilibria

\subsection{Numerical illustrations}

The previous subsection demonstrates the number and nature of Misspecification Equilibria in a New Keynesian model. To provide further results, this subsection presents numerical examples. We calibrate the model's parameters following Woodford (2003) as follows: $\varsigma=1 / 0.157, \kappa=0.024, \beta=0.99$. The weight in the forecast fitness measure $W$ is set to the identity matrix. Policy is assumed to satisfy the "Taylor principle" which states that nominal interest rates should be raised by more than one for one with inflation, in the case of a contemporaneous expectations rule the response coefficient is on expected inflation. In particular, we set $\chi_{\pi}=1.5, \chi_{x}=0.125$, which coincide with Taylor's original policy rule recommendation in Taylor (1993). In each example, we fix $\chi_{x}=0.125$. This subsection demonstrates that the model's equilibria will vary depending on the properties of the exogenous stochastic processes for $g_{t}, u_{t}$.

First, we demonstrate the possibility for multiple equilibria by setting $\rho=\phi=$ $0.5, \sigma_{\varepsilon}^{2}=0.9, \sigma_{v}^{2}=0.25, \sigma_{\varepsilon v}=0$. Figure 1 plots the T-map and relative predictor fitness measure $F(n)$ for various values of $n$.

The top panel of Fig. 1 plots the T-map while the bottom figure plots the relative profit fitness measure. Because the function $F$ is monotonic, so is the T-map. Moreover, the T-map crosses the 45-degree line three times, at $n=0, n=1$ and at a point $\hat{n}$ where $F(\hat{n})=0$. Clearly, the interior equilibrium has the property that $T^{\prime}(\hat{n})>1$ and so this equilibrium would not be attainable under a reasonable learning 

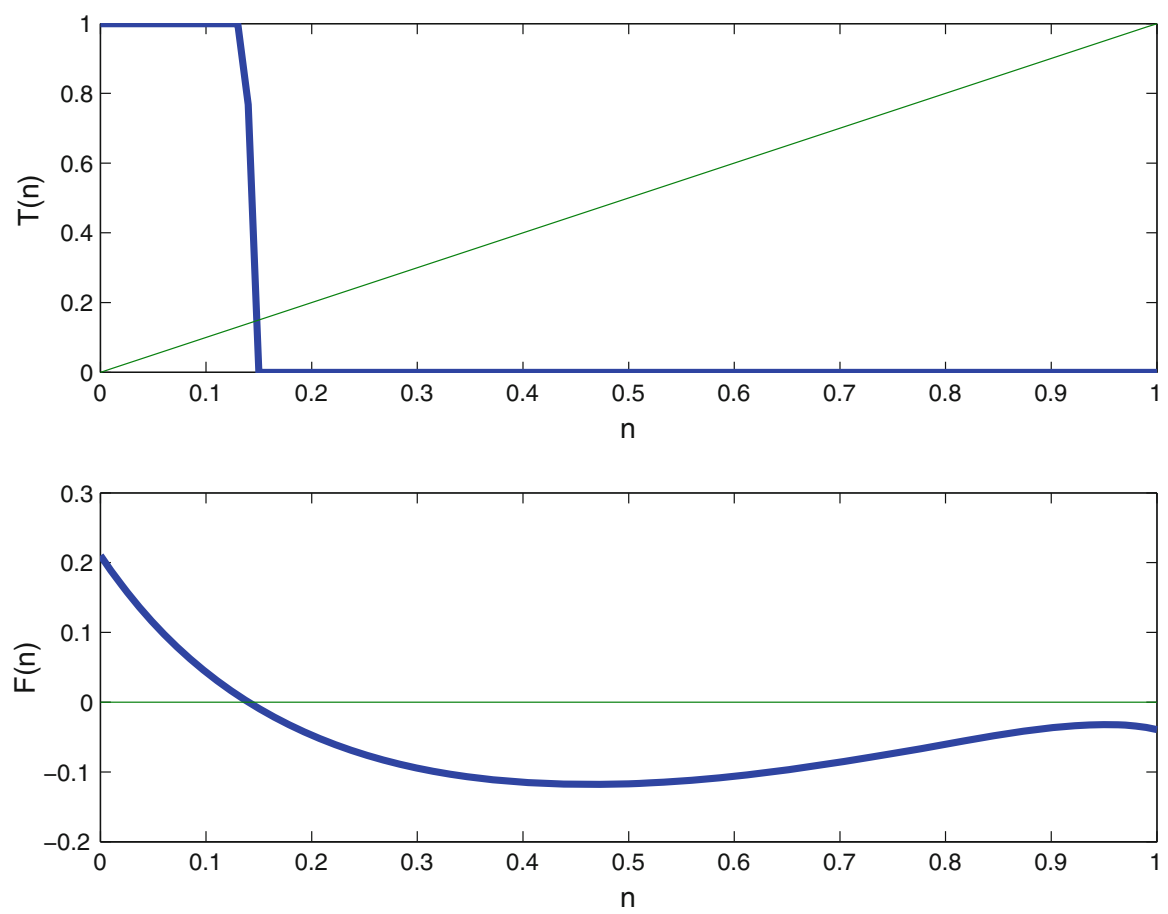

Fig. 2 Intrinsic heterogeneity

rule. However, the equilibria at $n=0$ and $n=1$ are stable, and so under this parameterization, with policy satisfying the Taylor principle multiple equilibria exist.

The results of the previous section, however, suggest that with alternative choices for the exogenous process governing demand and supply shocks that an equilibrium exhibiting Intrinsic Heterogeneity, where agents are distributed across all forecasting models, may exist. To illustrate this, we adopt the same parameter values as in Fig. 1, except now we set $\phi=.45, \sigma_{\varepsilon}^{2}=.35, \sigma_{v}^{2}=0.2$. Figure 2 plots the T-map and relative predictor fitness measure. The bottom panel shows that, under this particular parameterization, the function $F$ is no longer monotonic, and in particular it satisfies Condition $\mathrm{P}$ where $F(0)>0, F(1)<0$. Under Condition $\mathrm{P}$, equilibria where agents are all massed onto a particular forecasting model do not exist. The top panel illustrates that this is the case for these particular demand and price shock processes. Now the T-map is (monotonically) negatively sloped with a single interior fixed point. Hence, there is the possibility for diverse beliefs even though agents only select the best-performing forecasting models.

Figures 1 and 2 show that when policy satisfies the Taylor principle it is possible to have either multiple equilibria or Intrinsic Heterogeneity. It remains to be seen the role policy plays in the equilibrium properties of the model. To address this issue we turn to bifurcation diagrams. We adopt Woodford's calibration, make assumptions about the exogenous processes and the policy rule's response coefficient to output $\chi_{x}$, and then vary the inflation response coefficient $\chi_{\pi}>0$. For each value of $\chi_{\pi} \in[0,2]$, we 

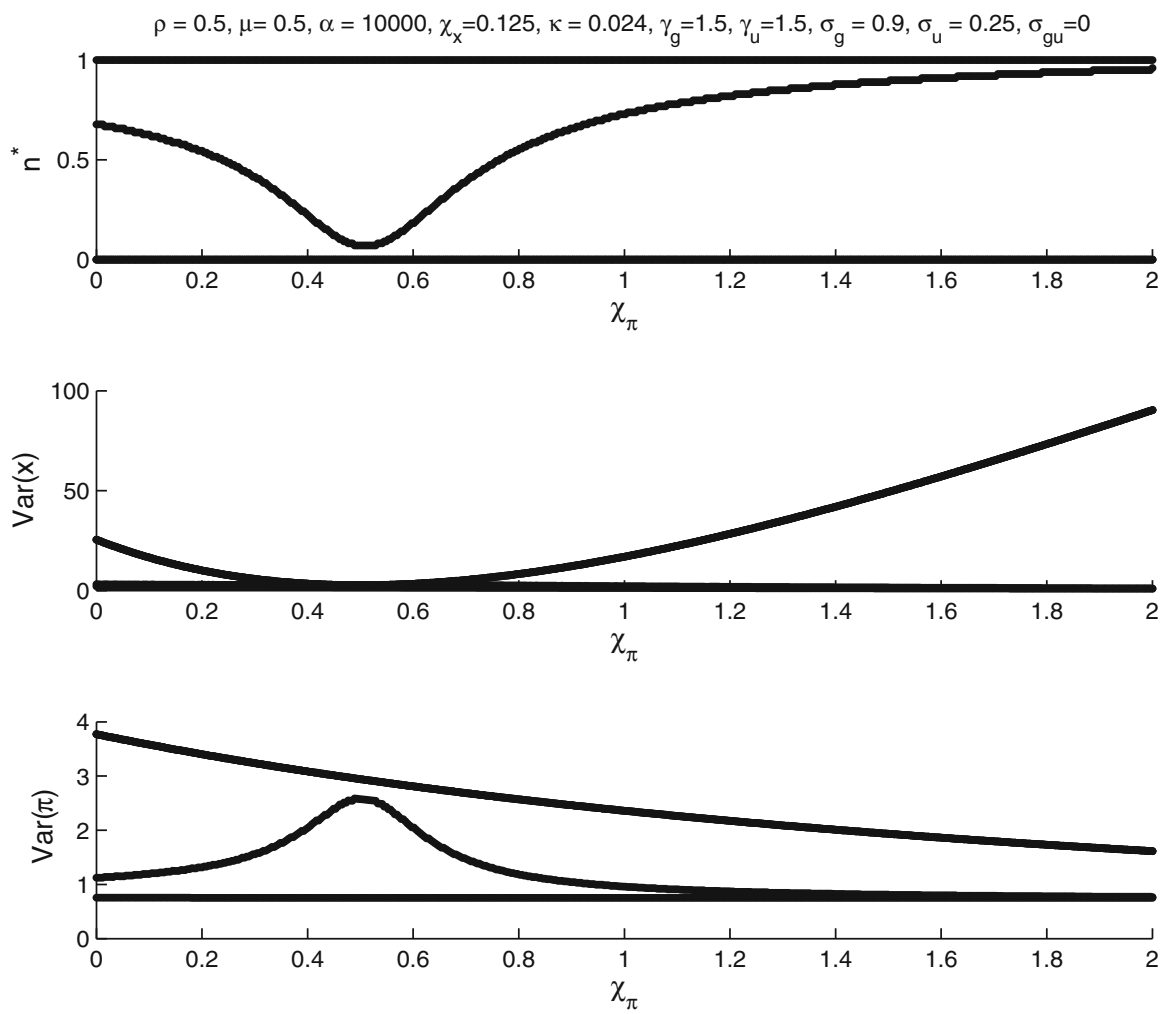

Fig. 3 Bifurcation diagram: multiple equilibria

calculate the fixed points $n^{*}=T\left(n^{*}\right)$, the corresponding unconditional variances of inflation and the output gap, and plot the results.

The top panel of Fig. 3 demonstrates that multiple equilibria exist for all values of the inflation response coefficient below two. As in Fig. 1 there are multiple equilibria at $\chi_{\pi}=1.5$. Although Fig. 3 shows the existence of multiple equilibria for all values of $\chi_{\pi}$ this does not imply that policy does not have an effect on the dynamic properties of the model. First, the top panel shows that the basins of attraction for the $n=0$ or $n=1$ equilibria vary with the policymaker's inflation response. If there were a real-time learning dynamic (see below) where agents select their forecasting model in real-time based on recursive estimates of the relative fitness of each model, then the size of the basin of attraction to equilibrium $n=1$ can be viewed as the vertical distance between the $n=1$ equilibrium and the unstable interior equilibrium. Clearly, at $\chi_{\pi} \approx 0.5$ the size of this basin is maximized. As $\chi_{\pi}$ increases further above one, the basin of attraction for the $n=1$ equilibria shrinks further and further. We know from Proposition 4 that for sufficiently large $\chi_{\pi}$ there is an equilibrium at $n=0$. So this basin of attraction for $n=1$ continues to shrink until the equilibrium disappears altogether.

The inflation response coefficient also affects the equilibrium variances for the economy. The bottom two panels plot the unconditional variances of output gap and inflation, respectively. In the middle panel, the equilibrium variance corresponding to 

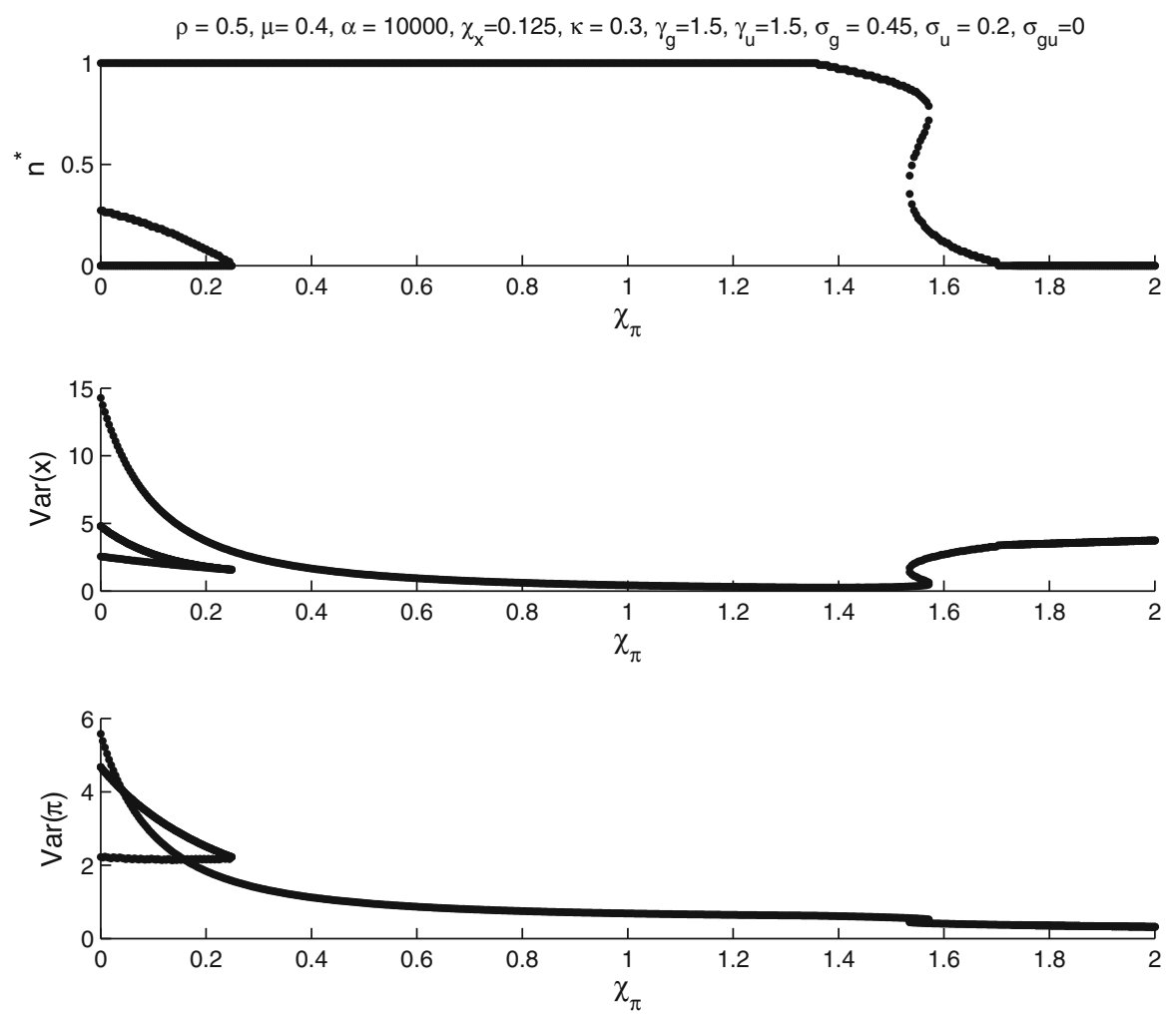

Fig. 4 Bifurcation diagram: intrinsic heterogeneity

$n=0$ starts off around 30, significantly above the variances for the $n=1$ and interior equilibrium, then approaches the values of the other equilibrium, before increasing sharply for $\chi_{\pi}$ above one. In the bottom panel, the smaller two variances correspond to the $n=1$ and $n=\hat{n}$ equilibria, while the higher line corresponds to the "supply shock" equilibrium $n=0$. These two panels demonstrate that for $\chi_{\pi}>0.5$ the $n=0$ equilibrium has the usual trade-off between inflation and output volatility. Moreover, it demonstrates that the equilibrium stochastic properties for the economy differ by equilibrium. Essentially, there is a low volatility $n=1$, "demand shock", equilibrium and a high volatility $n=0$ supply shock equilibrium. These results have important economic implications that we elaborate on further below.

Figure 4 demonstrates that some parameterizations can lead to a unique equilibrium with Intrinsic Heterogeneity. Here we adopt the same parameterization as above except now we set $\kappa=0.3, \sigma_{\varepsilon}^{2}=0.45$. Now for low values of $\chi_{\pi}$ we see as in Fig. 3 the existence of multiple equilibria: high volatility price shock equilibrium and low volatility demand shock equilibrium. However, for moderate inflation responses, including some that satisfy the Taylor principle, there exists a unique low volatility demand shock equilibrium. Eventually, for values of $\chi_{\pi}>1.4$ the $n=1$ equilibrium bifurcates and Intrinsic Heterogeneity becomes the unique equilibrium. Thus, under this parameterization, the standard Taylor rule coefficients would lead to diverse beliefs in 

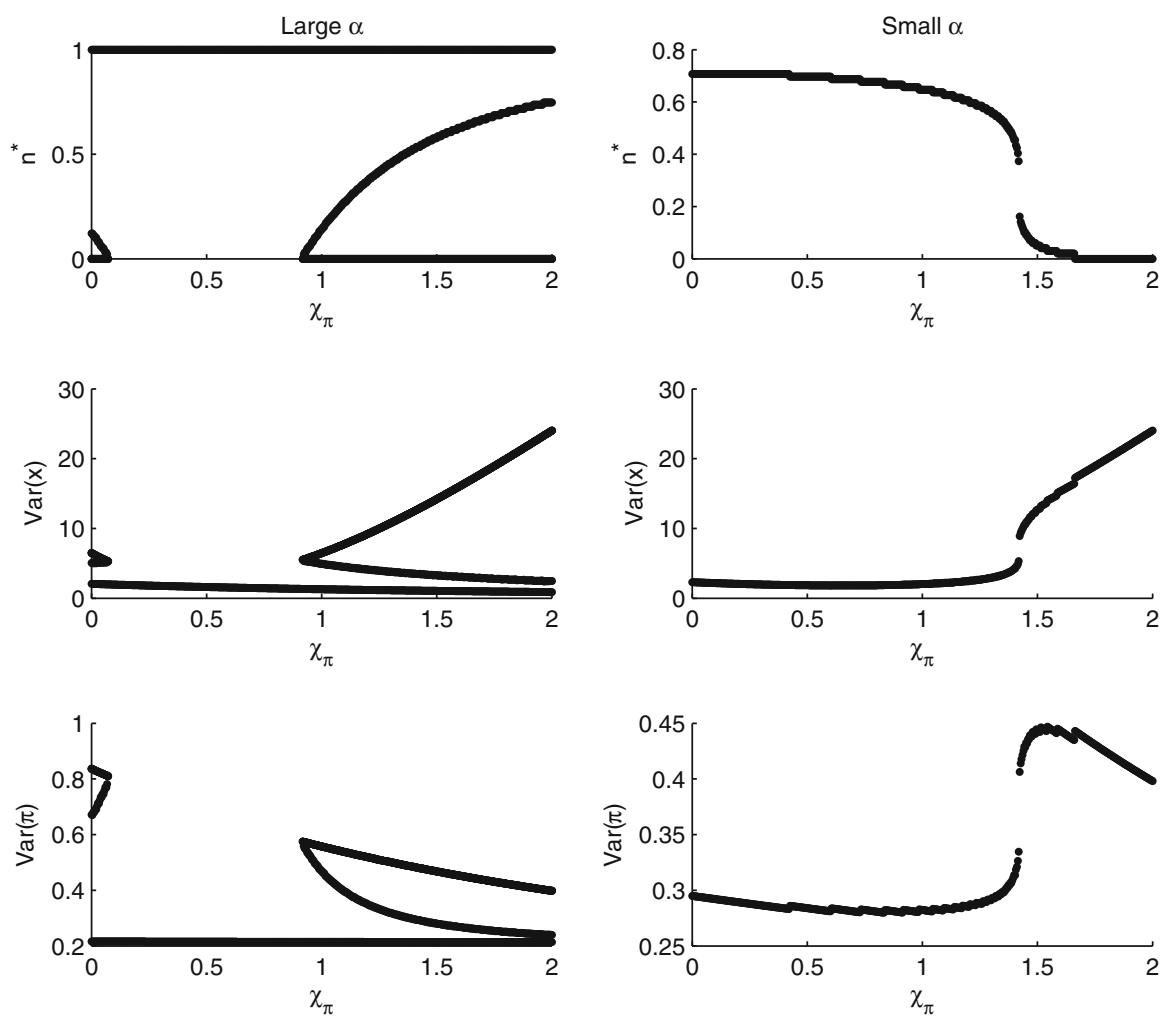

Fig. 5 Bifurcation diagram: large versus small $\alpha$

equilibrium. Eventually, though, as Proposition 4 shows, the price shock equilibrium emerges as the unique outcome.

So far we have been focusing on the neoclassical case of $\alpha=+\infty$. This case is appealing theoretically because all agents select only the best performing models. However, with finite $\alpha$ heterogeneity becomes a more pervasive feature of the economy, and there are good reasons to expect that agents will have finite intensities of choice. Young (2004) argues that a finite $\alpha$, which one can view as equivalent to having some randomness in predictor selection, is analogous to mixed strategies in game theory: with some uncertainty about the appropriate model agents can benefit by not being dogmatic in their choice of predictor. To see the impact that heterogeneity can have, we compare large and small $\alpha$ cases.

We first note that it is possible to have situations in which there are multiple equilibria with large $\alpha$, but a unique equilibrium, with diverse beliefs, for small $\alpha$. To demonstrate this, adopt the Woodford calibration and the parameter settings in Fig. 1, and set $\phi=0.45, \sigma_{v}^{2}=0.075$. Figure 5 plots the bifurcation diagram for various values of $\chi_{\pi}$ and for both large and small $\alpha$. The left plots show that with large $\alpha$ there can be multiple equilibria. The right plots illustrate our findings that when $\alpha$ takes a value less than or equal to three (the case $\alpha=3$ is shown), there exists a unique equilibrium with heterogeneous expectations. 

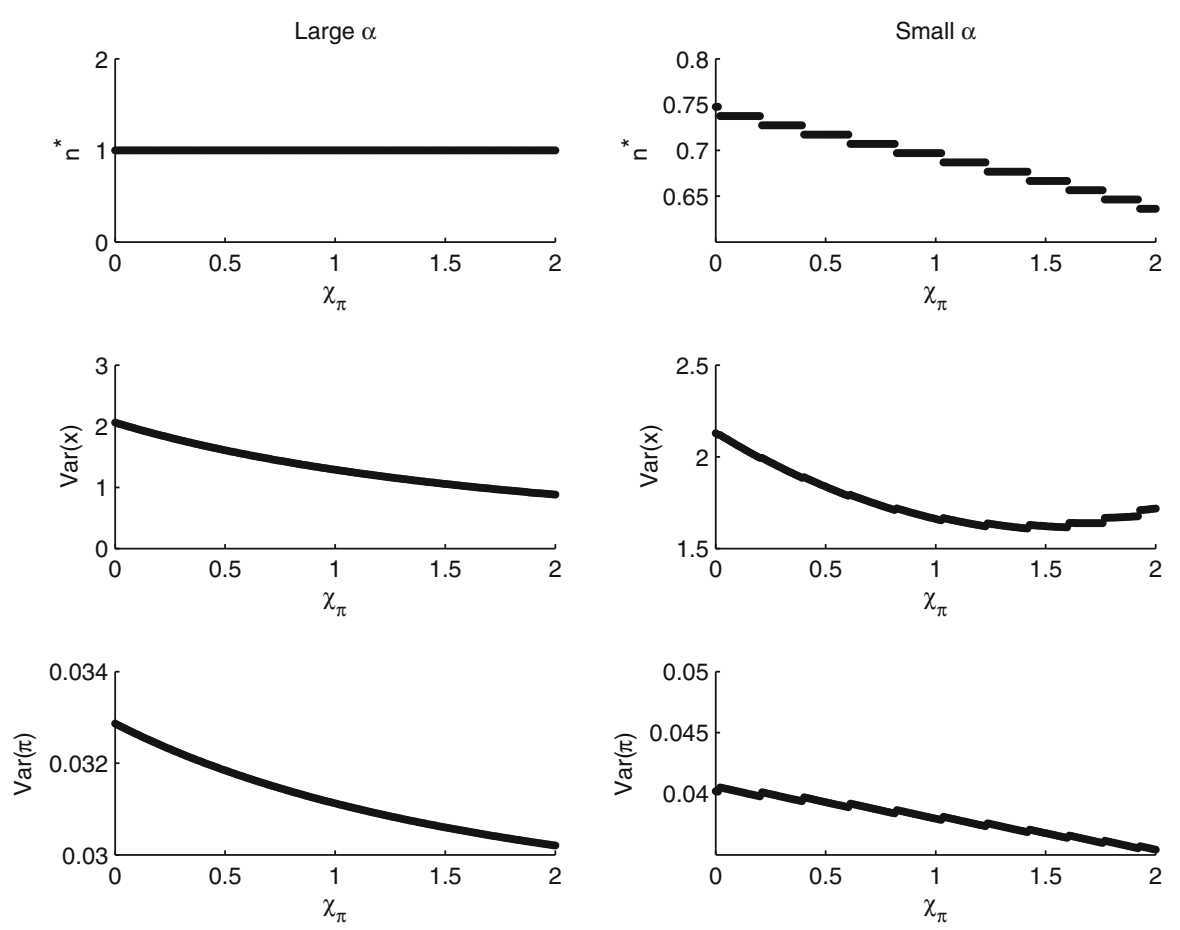

Fig. 6 Misspecification equilibria for large and small $\alpha$

Finite values of $\alpha$ can also qualitatively affect a model with a unique equilibrium under large $\alpha$. Figure 6 parameterizes the model as above except $\sigma_{v}^{2}=0.01$ so that there is a unique equilibrium at $n=1$ for large $\alpha$. With values of $\alpha<3$ there is a unique equilibrium with agents distributed across both forecasting models.

\section{Business cycle and policy implications}

Figures 3 and 4 have important business cycle and policy implications. This section demonstrates two results: a bad luck story, with regime-switching output and inflation variances, and the implications of heterogeneity for optimal monetary policy.

\subsection{Bad luck}

One widely cited empirical finding is that inflation and output volatility in the U.S., especially during the 1970's, follows a regime-switching process alternating between periods of high and low volatility (see Sims and Zha 2006). Stock and Watson (2003) present evidence that high inflation and output volatility during the 1970s coincided with a series of "bad luck" price shocks, e.g. high oil prices. This subsection investigates whether bad luck, in the form of greater persistence and volatility of price 

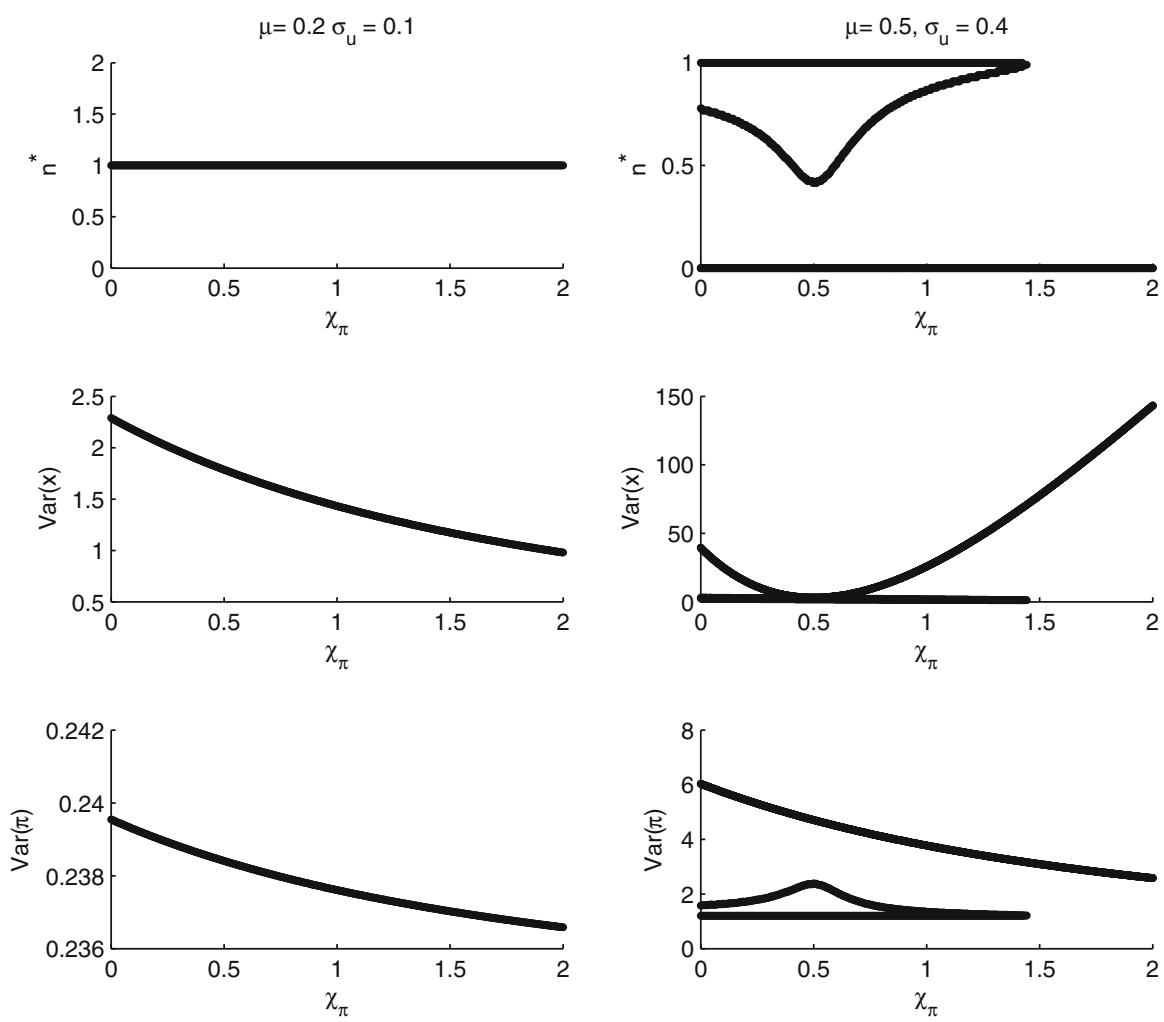

Fig. 7 Bifurcation diagram: bad luck

shocks, might lead to multiple equilibria and, under real-time learning and dynamic predictor selection, endogenous volatility.

We consider the following experiment. We parameterize the model as in Fig. 1, except that we set $\phi=0.2$ and $\sigma_{v}^{2}=0.1$. Then we imagine a "bad luck" structural change in which the persistence and variance of the price shock $u_{t}$ increases via the new parameter values $\phi=0.5$ and $\sigma_{v}^{2}=0.4$. For each of these two scenarios, Fig. 7 exhibits the equilibrium properties for various values of the inflation response coefficient $\chi_{\pi}$. The left-most panels of Fig. 7 arise under the case of low price shock variance while the right-most panels occur under the bad luck scenario. When price shocks have low persistence and volatility, there is a unique equilibrium at $n=1$ for all values of $\chi_{\pi}$. If, on the other hand, there is an episode of bad luck then there are multiple equilibria for values of $\chi_{\pi}<1.5$. The bad luck economy, therefore, could be in either a low volatility demand shock equilibrium or a high volatility price shock equilibrium.

Figure 7 also shows that if policy is sufficiently aggressive in response to inflation, i.e. $\chi_{\pi}>1.5$, the economy with more volatile price shocks will possess a unique equilibrium. This figure, therefore, captures two empirical features of the U.S. economy. First, bad luck can de-stabilize the economy in the sense that before the increase 
in the volatility of price shocks there was a unique equilibrium and afterwards there are multiple equilibria. Second, a monetary policy that responds strongly to expected inflation is stabilizing by coordinating the economy on a unique equilibrium. This second feature of Fig. 7, the stabilizing effect of anti-inflationary policy, has often been argued as a key component of the lower volatility during the mid-1980s-1990s. The model here explains these phenomena by highlighting the role that monetary policy and the stochastic properties of price shocks play as bifurcation parameters.

In Fig. 7, the policymaker can ensure existence of a unique equilibrium at $n=0$ by making policy sufficiently aggressive against expected inflation. However, in this particular numerical example stabilizing inflation comes at the expense of greater output volatility. A policymaker who instead increases the response of nominal interest rates to both inflation and output following a bad-luck episode can lead to reasonable variance outcomes for both inflation and output volatility. For example, following the bad luck episode in Fig. 7 by setting $\chi_{\pi}=1.5, \chi_{x}=1.5$ there is a unique equilibrium at $n=0$ and $\operatorname{Var}(x)=\operatorname{Var}(\pi)=4.5$.

The existence of multiple equilibria following a bad luck episode suggests that under a real-time learning and dynamic predictor selection formulation of the model, regime-switching output and inflation volatility might arise endogenously. We examine this possibility by turning to a real-time learning environment. In a Misspecification Equilibrium, agents' expectations satisfy the least-squares orthogonality conditions, where the expectation is taken with respect to population moments. We now assume that rather than knowing these population moments, and hence the equilibrium values for $b^{1}, b^{2}$ and the relative predictor fitness measure $F$, private-sector agents infer their values in real-time from historical data. In particular, we assume that agents use recursive least-squares to generate parameter estimates $b^{1}, b^{2}$ by regressing the state $y_{t}$ on demand or price shocks, respectively. In deciding on which forecasting model to adopt, they must also estimate the unconditional mean-square forecast errors recursively and select the model that delivers the lowest estimated squared forecast error.

Under real-time learning, the economy is generated by an actual law of motion with time-varying parameters:

$$
y_{t}=\xi_{1}\left(b_{t-1}^{1}, n_{t-1}\right) g_{t}+\xi_{2}\left(b_{t-1}^{2}, n_{t-1}\right) u_{t}
$$

where $b_{t}^{1}, b_{t}^{2}$ are updated by recursive least squares

$$
\begin{aligned}
b_{t}^{1} & =b_{t-1}^{1}+\eta_{t} R_{1 t}^{-1} g_{t}\left(y_{t}-b_{t-1}^{1} g_{t}\right) \\
b_{t}^{2} & =b_{t-1}^{2}+\eta_{t} R_{2 t}^{-1} u_{t}\left(y_{t}-b_{t-1}^{2} u_{t}\right) \\
R_{1 t} & =R_{1 t-1}+\eta_{t}\left(g_{t}^{2}-R_{1 t-1}\right) \\
R_{2 t} & =R_{2 t-1}+\eta_{t}\left(u_{t}^{2}-R_{2 t-1}\right) .
\end{aligned}
$$

$\eta_{t}$ is a deterministic gain sequence such that $\sum_{t=0}^{\infty} \eta_{t}=+\infty$. Under recursive least squares $\eta_{t}=t^{-1}$ is a decreasing gain and it is possible in many settings, including 
ours, to show that parameter estimates converge (with probability 1 ) to their equilibrium values. For the particular experiment under consideration, the $n=0, n=1$ restricted perceptions equilibria are locally stable under a decreasing gain learning rule. Alternatively, $\eta_{t}=\eta$, with $0<\eta<1$, is a constant gain version that assumes agents discount past data. A constant gain learning rule is desirable in environments in which agents may be concerned about structural change. After a bad luck structural change the economy may be in one of multiple equilibria, so an agent learning about the parameters of their forecasting rule(s) would want to use a constant gain algorithm to remain robust to the possibility of switching between equilibria.

In order to select a particular, recursively updated forecasting model, agents also estimate the unconditional mean square forecast error for each model,

$$
\begin{aligned}
& E U_{t}^{1}=E U_{t-1}^{1}+\delta_{t}\left[\left(y_{t}-b_{t-1}^{1} \rho g_{t}\right)^{2}-E U_{t-1}^{1}\right] \\
& E U_{t}^{2}=E U_{t-1}^{2}+\delta_{t}\left[\left(y_{t}-b_{t-1}^{2} \phi u_{t}\right)^{2}-E U_{t-1}^{2}\right]
\end{aligned}
$$

where $\delta_{t}$ is a gain sequence. Following Branch and Evans (2007) we allow $\delta_{t} \neq \eta_{t}$ so that agents may be more or less concerned with structural change in predictor fitness than in structural change in model parameters.

To illustrate the real-time learning and dynamic predictor selection dynamics we turn to numerical simulations. We set $\eta_{t}=0.03, \delta_{t}=0.05$, and adopt the parameter values in Fig. 7. We first initialize the model by simulating for 5000 periods, with a decreasing gain, allowing the parameters to converge to their $n=1$ equilibrium values. Then we simulate the model for 8000 periods. The first 4000 periods the economy will be in a good luck scenario with $\phi=0.2, \sigma_{v}^{2}=0.1$. Then at period 4001 the economy experiences a bad luck structural change with $\phi=0.5, \sigma_{v}^{2}=0.4$. Figure 8 plots a typical simulation.

The top panel of Fig. 8 plots the predictor selection in real-time. Prior to period 4000 there is a unique equilibrium, and so even under real-time learning agents coordinate on the demand shock equilibrium. Following period 4000 there are multiple equilibria, and the dynamics of predictor selection switch between the demand and supply shock equilibria as shocks to the system, filtered through agents' learning process, switch the economy between basins of attraction.

The bottom two panels of Fig. 8 plots a 20 quarter moving average of inflation and output volatility. Prior to period 4000 inflation and output variance are stable and near their low volatility $n=1$ equilibrium values. Following period 4000, as the economy switches between demand and supply shock equilibria, output and inflation variances fluctuate endogenously between high and low states.

The results in Fig. 8 suggest one (possible) interpretation of the high volatility periods in the U.S. as identified by Sims and Zha (2006). It has been argued, by Stock and Watson (2003) among many others, that the bad luck supply shocks of the 1970s can account for the high volatility of the 1970s and the subsequent period of moderation following 1984. However, the standard New Keynesian model cannot account for regime-switching volatilities unless the supply shock itself is assumed to 

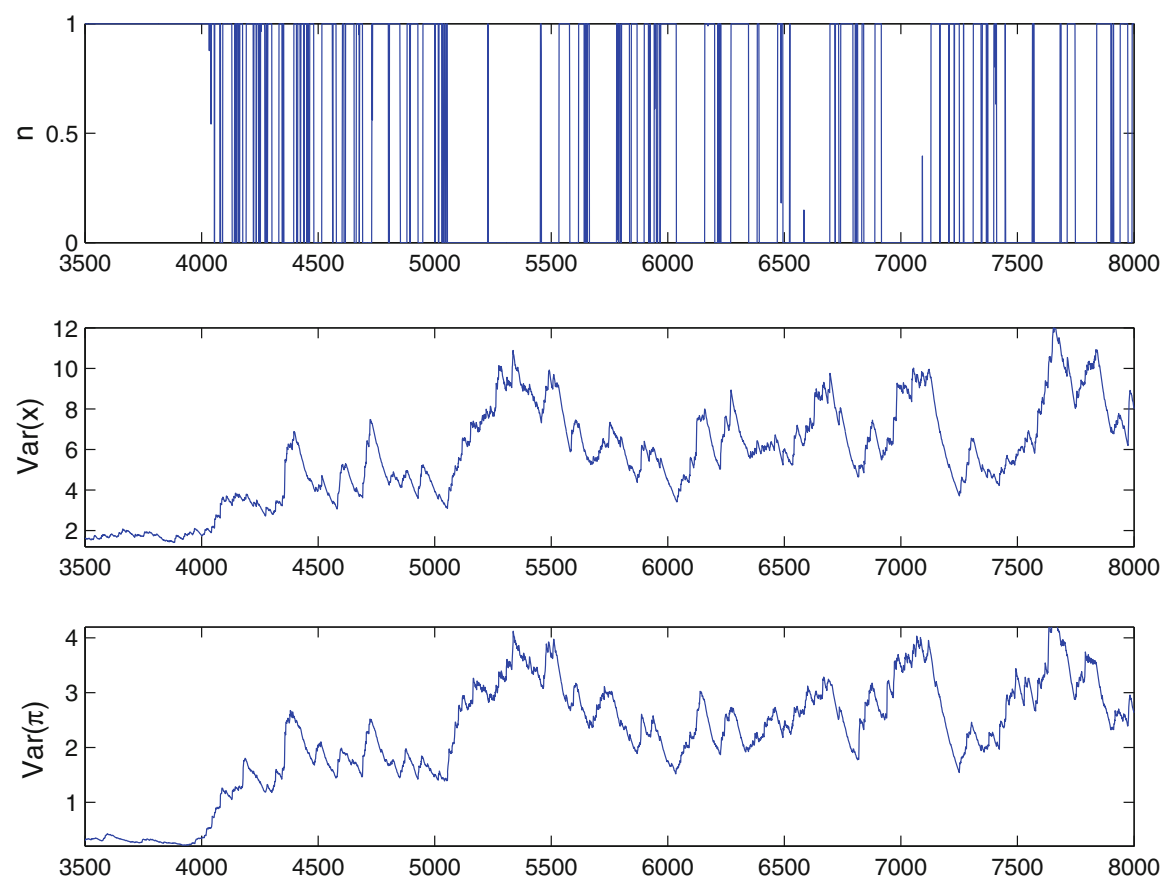

Fig. 8 Bad luck and endogenous volatility

follow a regime-switching process. Figure 8 demonstrates that a standard New Keynesian model adapted to incorporate a very reasonable model of expectation formation, can lead to multiple equilibria when supply shocks shift to a new higher variance process. The greater volatility of supply shocks has the indirect effect of leading to the existence of multiple equilibria. Under real-time learning, as agents update model parameters and select their models, the economy switches between high and low volatility equilibria. Our account thus provides a bad luck explanation for endogenous volatility. It is important to note that this endogenous volatility arises regardless of whether policy satisfies the Taylor principle. Figure 7 shows that in the bad luck scenario, multiple equilibria exist both for $\chi_{\pi}$ below and above one.

\subsection{A calibrated example}

The previous subsection demonstrated that a bad luck episode can lead the economy from a period of relative stability to unstable regime-switching dynamics. To further elaborate on the model's potential business cycle implications, this subsection presents a brief calibration exercise. We choose values for the policy parameters and shock processes in line with empirical estimates, simulate the model, and compare the moments of the model to U.S. data.

An empirical finding that we seek to replicate is that U.S. business cycle dynamics exhibit multiple regimes. As mentioned above, some attribute these regimes to changes 
Table 1 Business cycle calibration

\begin{tabular}{lllll}
\hline & $n=0$ & $n=1$ & $1960.1-1983.4$ & $1984.1-2009.2$ \\
\hline$\sigma_{y}$ & 2.32 & 1.04 & 2.00 & 0.932 \\
$\sigma_{\pi}$ & 0.70 & 0.429 & 0.91 & 0.25 \\
Inflation persistence & 0.37 & 0.38 & 0.92 & 0.45 \\
corr $(y, \pi)$ & -0.745 & 0.081 & 0.012 & 0.26 \\
\hline
\end{tabular}

Standard deviation in percent of log real GDP, $y$, and inflation is calculated from the implicit price deflator. Data have been HP-detrended

in the conduct and goals of monetary policy (e.g. Clarida et al. 1999) and others to changes in the underlying structural shocks (e.g. Stock and Watson 2003). The results from the previous section suggest that regime-switching can arise from a model in which agents select underparameterized forecasting models even if there are no structural changes to policy or the shocks. This subsection explores to what extent learning and dynamic predictor selection, without changes in monetary policy or structural features of the economy, can be a plausible explanation for regime-switching business cycle dynamics.

We adopt the Woodford calibration assumed throughout the paper and additionally set $\chi_{\pi}=1.01, \chi_{x}=.125, \rho=.5, \phi=.45, \sigma_{\varepsilon}=0.9, \sigma_{v}=0.075$. These parameter values are consistent with empirical estimates presented in the literature. There is some evidence that $\chi_{\pi}<1$ during the 1970's and $\chi_{\pi}>1$ in subsequent periods. We set $\chi_{\pi}=1.01$ as a compromise between these findings and to be sure that the results are not being driven by a failure to satisfy the Taylor principle. Estimates for $\rho, \phi$ tend to be greater than these assumed values, but the calibrated values fall within a typical $90 \%$ confidence set.

We simulate the model with learning and dynamic predictor selection. We adopt the constant gains from the previous subsection: $\eta_{t}=0.03, \delta_{t}=0.05$. We first simulate the model for a transient 5000 periods, then simulate the model for 8000 periods, calculate moments from the simulated data, and then repeat 1000 times. We then average the moments across simulations. Table 1 presents the results.

In Table 1, we report on the standard deviations of output, inflation, the contemporaneous correlation of output and inflation, and the persistence of inflation by regime. The model parameterization chosen leads to the existence of multiple misspecification equilibria, with the $n=0, n=1$ equilibria stable under learning. With constant gain learning, the economy switches between these equilibria as in Fig. 8. We calculate the moments within each $n=1$ or $n=0$ regime.

Table 1 compares the moments from the model with those from the data. There have been several approaches to identifying regime change in U.S. data. One approach is to assume a regime change after Volcker is appointed chairman of the Federal Reserve, or at the time of the Great Moderation in 1984. We compare the moments from the model across the two $n=0, n=1$ regimes to the moments from U.S. data pre and post 1984.1. Similar results obtain for other approaches to identifying breaks in the data. For each series the data have been detrended using the HP filter.

Table 1 demonstrates that the relative moments across regimes for the model and for the data are an approximate match. In the data output and inflation volatility drop 
by 53 and $72 \%$ while the model implies a reduction in volatility of 55 and 39\%, respectively. Similarly, the model implies values for the contemporaneous correlation and inflation persistence for the $n=1$ regime very similar to that for the data. On the other hand, the model does not deliver as well for these variables in the $n=0$ regime. The negative contemporaneous correlation in the $n=0$ regime arises because in this case the economy is driven primarily by supply shocks.

That the results in this simple model, in which agents select predictors in real time, can deliver empirically plausible regime-switching dynamics, provides some support for the misspecification approach advocated for in this paper. These results arise in a calibrated model without change in the policy rule or the exogenous shocks. A more realistic model would build in switching policy rules and stochastic volatility in the exogenous shocks. We anticipate that results from such a model would improve the fit over the simple case presented in this section.

\subsection{Optimal discretionary policy}

Under a contemporaneous policy rule, there are many possible equilibrium outcomes. A natural question then is what if policymakers were to conduct optimal discretionary policy? To address this question, suppose that policymaker's seek to minimize a quadratic loss function,

$$
E_{0} \sum_{t \geq 0} \beta^{t}\left(\omega x_{t}^{2}+\pi_{t}^{2}\right)
$$

subject to the aggregate supply curve (2). Under discretion, it is possible to find a nominal interest rate rule that implements the optimal discretionary policy and responds only to the fundamental shocks. This fundamentals based rule was shown by Evans and Honkapohja (2003) to lead to an unstable (and indeterminate) rational expectations equilibrium. Under underparameterized beliefs it is possible to show that the restricted perceptions equilibrium is also unstable when policy is formed via a fundamentalsbased rule. Instead, Evans and Honkapohja (2003) propose a rule that responds to private sector expectations and the fundamental exogenous shocks. When monetary policy responds directly to private-sector expectations, under least-squares learning the temporary equilibrium outcomes will converge to a unique rational expectations equilibrium. The good economic properties of an expectations-based rule have been established under the assumption of homogeneous expectations. This subsection considers the properties of expectations-based rules under heterogeneous expectations.

It is possible to show that a policy rule of the form

$$
i_{t}=\varsigma^{-1} \hat{E} x_{t+1}+\left(1+\frac{\beta \kappa \varsigma^{-1}}{\omega+\kappa^{2}}\right) \hat{E}_{t} \pi_{t+1}+\frac{\kappa \varsigma^{-1}}{\omega+\kappa^{2}} u_{t}+\varsigma^{-1} g_{t}
$$

will implement optimal discretionary policy under misspecified beliefs. Furthermore, taking the same parameterization as in Fig. 1, Fig. 9 plots the T-map. The left-hand plots of Fig. 9 give the case of the EH-rule that implements optimal discretionary 

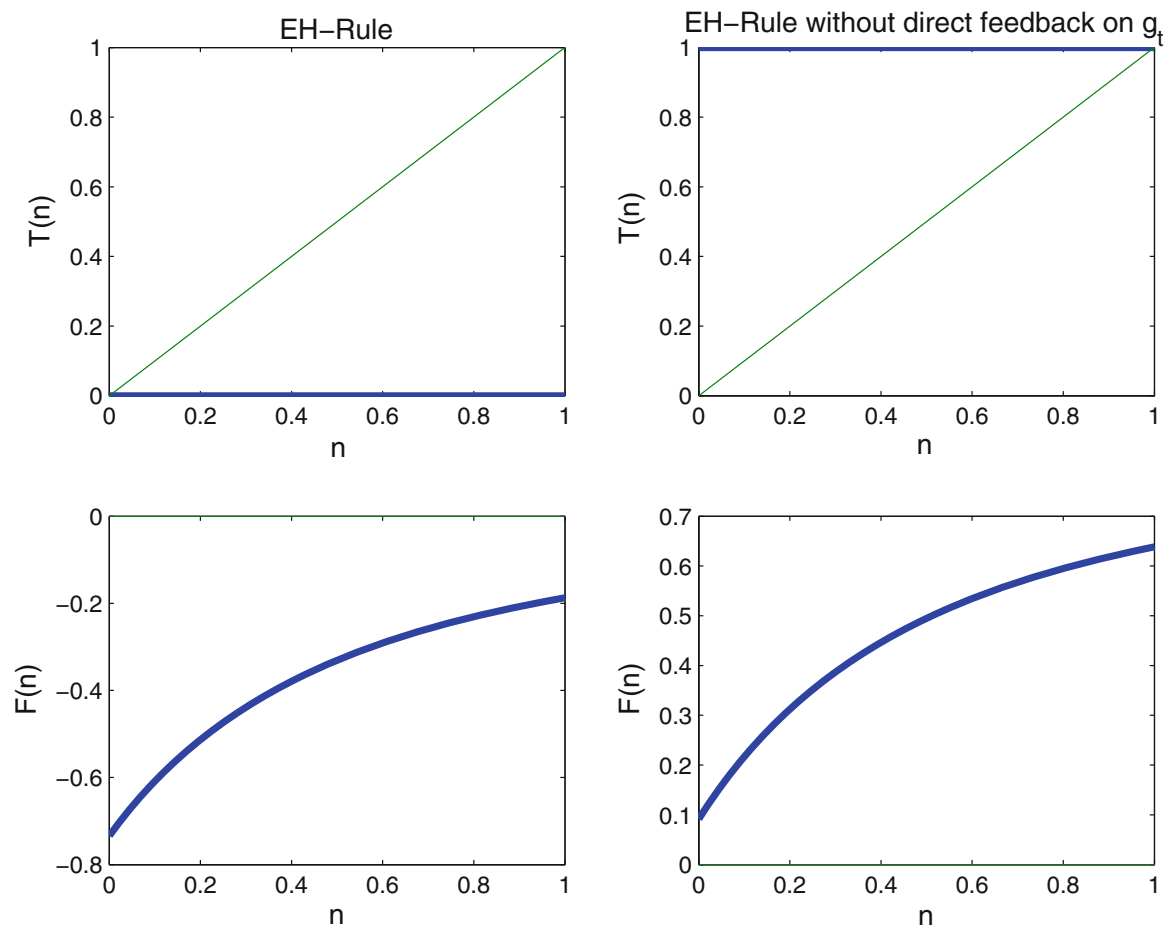

Fig. 9 Optimal discretionary policy and expectations-based rules

policy. This figure makes clear that the optimal policy rule coordinates the economy on the $n=0$ equilibrium. This result is expected because optimal policy perfectly offsets demand shocks, leaving the economy only to depend on price shocks. Because the economy is driven entirely by price shocks there is only one possible equilibrium outcome: $n=0$. Moreover, this equilibrium coincides with the optimal discretionary rational expectations equilibrium.

The right-most plots consider the same parameter values, the same expectations based rule, but where the policymakers do not directly respond to demand shocks $g_{t}$. In this case, there is a unique equilibrium where agents coordinate on the demand shock model.

\subsection{Discussion of related literature}

Although most monetary models rely on a representative agent structure, this paper demonstrates that there are important implications for monetary policy and business cycle dynamics from a model with heterogeneous expectations. Heterogeneity arises in our model as the result of the interaction between the direct effect of shocks, the indirect effect that arises through the self-referential features of the New Keynesian model, and the policy feedback effect. 
The endogeneity of heterogeneous beliefs is an important feature in other models as well. For example, Branch and McGough (2011) study a neoclassical stochastic growth model with agents distributed heterogeneously between rational and adaptive agents, whose beliefs are pinned down by a restricted perceptions equilibrium. Kurz and Motolese (2010) and Guo et al. (2011) demonstrate that diverse beliefs can have important implications for asset pricing and asset returns. These latter two papers adopt the Rational Belief Equilibrium (RBE) approach of Kurz (1994). The RBE methodology emphasizes that beliefs are a component of the state of the economy, and in equilibrium the empirical distribution of the economy must align itself with the beliefs of the agents. In Kurz et al. (2005), it is shown that a model with RBE can significantly improve the empirical fit of monetary business cycle models.

Closely related to this paper is De Grauwe (2010) who introduces heterogeneous beliefs into a New Keynesian model. In his model, there are two types of agents, optimists and pessimists. The diversity of their opinion is increasing with the output gap. DeGrauwe shows that the positive feedback of the New Keynesian model will lead agents to coordinate on a particular model, leading to endogenous boom-bust dynamics. The importance of positive versus negative feedback for the way in which heterogeneous expectations arise in our setting has also been emphasized in our earlier work. In an eductive setting the closely related role of complementarity vs. substitutability is shown by Guesnerie and Jara-Moroni (2011) to play a pivotal role in expectational coordination. ${ }^{2}$

A key aspect to the present paper is that agents adopt heterogeneous forecasting models. We motivated this assumption by highlighting an empirical literature that finds evidence of heterogeneous expectations. Wieland and Wolters (2010) also demonstrates that the model implied forecasts can vary significantly from one estimated DSGE model to the next. It would be interesting for future research to consider misspecification equilibria when agents adopt forecasts from misspecified DSGE models. ${ }^{3}$

\section{Conclusion}

Most of the literature on monetary policy adheres to the assumption of rational expectations, in which agents have homogeneous expectations. Recent studies of the implications for monetary policy of private agents following adaptive learning rules have for the most part maintained the assumption of homogeneous expectations. However, we know from surveys that heterogeneous beliefs are a salient feature of the data. In our framework, heterogeneous expectations arise naturally because agents adopt one of several competing forecasting models, with the distribution of agents across predictors reflecting the relative success of the alternative forecast rules. In some cases heterogeneity exists even in the limit when agents select only the best predictors. This can arise in particular when monetary policy responds very aggressively to expected output or inflation.

\footnotetext{
2 This is also seen in the experimental results of Fehr and Tyran (2008).

3 For a related approach in an estimated DSGE model, see Slobodyan and Wouters (2007).
} 
Our framework has turned up new phenomena. Multiple equilibria is possible even when there is a unique rational expectations equilibrium that is stable under least-squares learning. In particular, bad luck in the form of greater persistence and volatility of price shocks, such as occurred in the 1970s, could push the economy from a unique equilibrium to a situation with multiple equilibria. Under real-time learning and dynamic predictor selection the economy would exhibit endogenous volatility. Increasing the policy response to inflation in the Taylor rule, as arguably occurred in the mid 1980s, can eliminate the endogenous volatility.

We also examined optimal discretionary monetary policy, looking in particular at an expectations based-rule known to have good properties under least-squares learning. We found that this rule, designed to respond explicitly to private-sector expectations, continues to perform well in our set-up in the presence of heterogenous expectations. It would be of interest to examine the generality of this finding and to extend the investigation to rules with history dependence of the type discussed in the literature on optimal policy with commitment.

\section{Appendix}

Proof of proposition 1 Computing the determinant and inserting $r=\tilde{r}=\rho=\phi=0$ we obtain

$$
\operatorname{det}(\Delta)=\left(1+\chi_{x}(1-n) \varsigma+\chi_{\pi}(1-n) \varsigma \kappa\right)\left(1+n \chi_{x} \varsigma+n \chi_{x} \varsigma \kappa\right) .
$$

Since $\operatorname{det}(\Delta) \geq 1$, the result follows by continuity.

Proof of proposition 3 The reduced-form matrix expressions for $A, B, D$ are

$$
\begin{aligned}
& A=\left(\begin{array}{cc}
1 & \varsigma \\
\kappa & \beta+\kappa \varsigma
\end{array}\right) \\
& B=\left(\begin{array}{cc}
-\chi_{x} \varsigma & -\chi_{\pi} \varsigma \\
-\chi_{x} \kappa \varsigma & -\chi_{\pi} \kappa \varsigma
\end{array}\right) \\
& D=\left(\begin{array}{ll}
1 & 0 \\
\kappa & 1
\end{array}\right) \text {. }
\end{aligned}
$$

Since $b^{1}=\xi_{1}$ and $b^{2}=\xi_{2}$ when $r, \tilde{r} \rightarrow 0$ it follows that $\xi_{1}(0)=(1, \kappa)^{\prime}$,

$$
\xi_{2}(0)=\left(\begin{array}{c}
\frac{\varsigma\left(\phi-\chi_{\pi}\right)}{1+\varsigma\left(\chi_{x}+\kappa \chi_{\pi}\right)-\phi\left(\left(1+\beta+\varsigma\left(\beta \chi_{x}+\kappa\right)+\beta \phi\right)\right.} \\
\frac{1+\varsigma \chi_{x}-\phi}{1+\varsigma\left(\chi_{x}+\kappa \chi_{\pi}\right)-\phi\left(\left(1+\beta+\varsigma\left(\beta \chi_{x}+\kappa\right)+\beta \phi\right)\right.}
\end{array}\right) .
$$

Similarly, $\xi_{2}(1)=(0,1)^{\prime}$ and

$$
\xi_{1}(1)=\left(\begin{array}{c}
\frac{1-\beta \rho}{1+\beta \rho^{2}+\varsigma\left(\chi_{x}+\kappa \chi_{\pi}\right)-\rho\left(1+\beta+\varsigma\left(\beta \chi_{x}+\kappa\right)\right)} \\
\frac{\kappa}{1+\beta \rho^{2}+\varsigma\left(\chi_{x}+\kappa \chi_{\pi}\right)-\rho\left(1+\beta+\varsigma\left(\beta \chi_{x}+\kappa\right)\right)}
\end{array}\right) .
$$


Evaluating $F(0)$ and $F(1)$ at the above values for $\xi_{1}, \xi_{2}$, it can be seen that

$$
\begin{aligned}
& F(0)<0 \Leftrightarrow \frac{\sigma_{v}^{2}}{\sigma_{\varepsilon}^{2}}>B_{0} \\
& F(1)<0 \Leftrightarrow \frac{\sigma_{v}^{2}}{\sigma_{\varepsilon}^{2}}>B_{1},
\end{aligned}
$$

from which the result follows.

Proof of proposition 4 For result 1, it is straightforward to see that $\lim _{\chi_{\pi} \rightarrow \infty} B_{1}=$ $\lim _{\chi_{x} \rightarrow \infty} B_{1}=0$. Moreover, using l'Hôpital's rule

$$
\begin{aligned}
& \lim _{\chi_{\pi} \rightarrow \infty} B_{0}=\frac{\rho^{2}\left(1-\phi^{2}\right)\left(1+\kappa^{2}\right) \kappa^{2}}{\phi^{2}\left(1-\rho^{2}\right)}, \text { and } \\
& \lim _{\chi_{x \rightarrow \infty}} B_{0}=\frac{\rho^{2}\left(1-\phi^{2}\right)\left(1+\kappa^{2}\right)(1-\beta \phi)^{2}}{\phi^{2}\left(1-\rho^{2}\right)} .
\end{aligned}
$$

The result follows. Result 2 follows since $P 0$ must hold for $\frac{\sigma_{v}^{2}}{\sigma_{\varepsilon}^{2}}$ sufficiently large. Similarly Result 3 follows since $P 1$ must hold for $\frac{\sigma_{v}^{2}}{\sigma_{\varepsilon}^{2}}$ sufficiently small.

Acknowledgments The second author acknowledges support from the National Science Foundation.

Open Access This article is distributed under the terms of the Creative Commons Attribution Noncommercial License which permits any noncommercial use, distribution, and reproduction in any medium, provided the original author(s) and source are credited.

\section{References}

Branch, W.A.: The theory of rationally heterogeneous expectations: Evidence from survey data on inflation expectations. Econ J 114, 592-621 (2004)

Branch, W.A., Evans, G.W.: Intrinsic heterogeneity in expectation formation. J Econ Theory 127, 264295 (2006)

Branch, W.A., Evans, G.W.: Model uncertainty and endogenous volatility. Rev Econ Dyn 10, 207237 (2007)

Branch, W.A., Evans, G.W.: Asset return dynamics and learning. Rev Financial Stud (2009, forthcoming)

Branch, W.A., McGough, B.: A New Keynesian model with heterogeneous expectations. J Econ Dyn Control 33, 1036-1051 (2009)

Branch, W.A., McGough, B.: Business cycle amplification with heterogeneous expectations. Econ Theory, this volume (2010)

Brock, W.A., Hommes, C.H.: A rational route to randomness. Econometrica 65, 1059-1095 (1997)

Bullard, J., Mitra, K.: Learning about monetary policy rules. J Monet Econ 49, 1105-1129 (2002)

Clarida, R., Gali, J., Gertler, M.: The science of monetary policy: a New Keynesian perspective. J Econ Lit 37, 1661-1707 (1999)

De Grauwe, P.: Animal spirits and monetary policy. Econ Theory, this volume (2010)

Evans, G.W., Honkapohja, S.: Learning and Expectations in Macroeconomics. Princeton: Princeton University Press (2001)

Evans, G.W., Honkapohja, S.: Expectations and the stability problem for optimal monetary policies. Rev Econ Stud 70, 807-824 (2003) 
Fehr, E., Tyran, J.R.: Limited rationality and strategic interaction: the impact of the strategic environment on nominal inertia. Econometrica 76, 353-394 (2008)

Guesnerie, R., Jara-Moroni P.: Expectational coordination in a class economies with strategic substitutabilities: the complete information case. Econ Theory, this volume (2010)

Guo, W.C., Wang, F.Y., Wu, H.M.: Financial leverage and market volatility with rational beliefs. Econ Theory, this volume (2010)

Kurz, M.: On the structure and diversity of rational beliefs. Econ Theory 4, 1-24 (1994)

Kurz, M., Jin, H., Motolese, M.: The role of expectations in economic fluctuations and the efficacy of monetary policy. J Econ Dyn Control 29, 2017-2065 (2005)

Kurz, M., Motolese, M.: Diverse beliefs and time variability of risk premia. Econ Theory, this volume (2010)

McCallum, B.T.: Issues in the design of monetary policy rules, Chapter 23, pp. 1483-1530. See Taylor and Woodford (1999)

Sargent, T.J.: The Conquest of American Inflation. Princeton University Press: Princeton (1999)

Sargent, T.J.: Evolution and intelligent design. Am Econ Rev 98, 5-37 (2008)

Sims, C., Zha, T.: Were there regime switches in us monetary policy? Am Econ Rev 96, 54-81 (2006)

Slobodyan, S., Wouters, R.: Learning in an estimated DSGE model. mimeo (2007)

Stock, J.H., Watson, M.W.: Has the business cycle changed? evidence and explanation. In: Monetary Policy and Uncertainty: Adapting to a Changing Economy. Proceedings of symposium sponsored by Federal Reserve Bank of Kansas City, Jackson Hole, WY (2003)

Taylor, J.: Discretion versus policy rules in practice. Carnegie-Rochester Conference Series in Public Policy 39, 195-214 (1993)

Taylor, J., Woodford M., (Eds): Handbook of Macroeconomics, vol. 1. Amsterdam, Elsevier (1999)

Wieland, V., Wolters, M.: The diversity of forecasts from macroeconomic models of the U.S. economy. Econ Theory, this volume (2010)

Woodford, M.: Interest and Prices: Foundations of a Theory of Monetary Policy. Princeton: Princeton University Press (2003)

Young, P.H.: Strategic Learning and its Limits. University Press: Oxford (2004) 